\title{
Adaptive immunity-related gene expression profile is correlated with clinical phenotype in patients with acute myeloid leukemia
}

\author{
Peng Chen ${ }^{1 \#}$, Yi Liu ${ }^{1 \#}$, Rui Zhang ${ }^{2}$, Haitao Wang ${ }^{1}$, Juan Zhang ${ }^{1}$, Meng Guo ${ }^{3}$, Zhenlan Du ${ }^{4,5,6,7}$ ^ \\ ${ }^{1}$ Department of Hematology, The Fifth Medical Center of Chinese PLA General Hospital, Beijing, China; ${ }^{2}$ Department of Hematology, People's \\ Hospital of Cangzhou, Cangzhou, China; ${ }^{3}$ Xijing Hospital of Digestive Diseases, Air Force Medical University (Fourth Military Medical University), \\ Xi'an, China; ${ }^{4}$ Department of Hematology and Oncology, Faculty of Pediatrics, Chinese PLA General Hospital, Beijing, China; ${ }^{5}$ Bayi Children's \\ Hospital, Seventh Medical Center of Chinese PLA General Hospital, Beijing, China; ${ }^{6}$ National Engineering Laboratory for Birth Defects Prevention \\ and Control of Key Technology, Beijing, China; ${ }^{7}$ Beijing Key Laboratory of Pediatric Organ Failure, Beijing, China \\ Contributions: (I) Conception and design: Z Du; (II) Administrative support: J Zhang; (III) Provision of study materials or patients: H Wang; (IV) \\ Collection and assembly of data: R Zhang; (V) Data analysis and interpretation: P Chen; Y Liu; M Guo; (VI) Manuscript writing: All authors; (VII) \\ Final approval of manuscript: All authors. \\ \#These authors contributed equally to this work. \\ Correspondence to: Zhenlan Du. Department of hematology and oncology, Faculty of Pediatrics, Chinese PLA General Hospital, Beijing, China; Bayi \\ Children's Hospital, Seventh Medical Center of Chinese PLA General Hospital, Beijing, China; National Engineering Laboratory for Birth Defects \\ Prevention and Control of Key Technology, Beijing, China; Beijing Key Laboratory of Pediatric Organ Failure, Beijing, China. Email: duzlan@163.com.
}

Background: Acute myeloid leukemia (AML) is a common and lethal hematopoietic malignancy that is highly dependent on the immune microenvironment. However, light has yet to be shed on the landscape of adaptive immunity-related genes. This work aimed to uncover the novel molecular events in AML and potential therapeutic strategies for AML treatment.

Methods: For the current research, the transcriptional information of 732 genes that participate in adaptive immunity was collected from 173 patients with AML, and the patients were grouped into different cohorts based on the different expression patterns. The correlations between gene expression and clinical characteristics, including prognosis, were studied.

Results: According to the notably different expressions of adaptive immunity-related genes, the 173 patients were divided into 2 clusters and 3 subclusters. No significant differences in overall survival (OS) or progression-free survival (PFS) were detected between the clusters or subclusters. There were obvious discrepancies found in age, peripheral blood (PB) blast percentage, and French-American-British (FAB) classification between each cluster or subcluster. The patients in cluster 1 were older and more of them had M5 type; the patients in cluster 2 were younger and more of them had M2 type. Further, 81 genes were significantly correlated with age and 101 genes were significantly correlated with PB blast percentage. Comparison of the prognosis between each FAB type revealed that patients with M3 type displayed the most favorable OS and PFS. Among the differentially expressed genes (DEGs), CLEC2B expression was much lower in M2 patients than in patients with other types $(\mathrm{P}<0.001)$, and its high expression indicated a worse outcome (12.4 vs. 46.5 months of OS).

Conclusions: This study has uncovered the expression profile of adaptive immunity-related genes in AML. The different gene expression patterns are not associated with survival, but are significantly correlated the FAB types. CLEC2B expression is low in patients with M2 type and is negatively correlated with prognosis, thus revealing a potential therapeutic target for AML.

\footnotetext{
^ ORCID: 0000-0002-3130-7780.
} 
Keywords: Acute myeloid leukemia (AML); adaptive immunity; French-American-British (FAB) subtypes; prognosis

Submitted Apr 06, 2021. Accepted for publication Jun 09, 2021.

doi: $10.21037 / \mathrm{atm}-21-2720$

View this article at: http://dx.doi.org/10.21037/atm-21-2720

\section{Introduction}

Acute myeloid leukemia (AML) is a genetically heterogeneous disease which is characterized by abnormal hematopoiesis caused by the accumulation and expansion of immature myeloid cells in the bone marrow and peripheral blood (PB) (1). Although chemotherapy can prolong the prognosis of AML, most patients suffer relapse (2). Allogeneic transplantation is an effective therapy for AML, but relapse after transplantation occurs commonly, leading to a dismal outcome (3). Progress in the genetic investigation of AML has seen researchers excavate the etiology of leukemogenesis, which has promoted the development of potential novel targeted therapies (4). Hematopoietic cell-specific immune molecules, like CD33, have been found to be promising and ideal targets for AML treatment $(5,6)$. Previous studies have revealed that the aberrant expression of immunity-related genes is closely associated with clinical outcome in patients with AML $(7,8)$. Therefore, a comprehensive understanding of immunerelated genes and molecular genetic characteristics is essential for diagnosis and outcome prediction in AML.

In multiple tumors, the immune system can identify and eliminate cancerous cells in a process termed cancer immunosurveillance. However, some studies have evidenced that the immune system can also promote tumor progression (9). In humans, innate immunity constitutes the primary facility of host defense against various anomalies, and it also guides the adaptive immune response (10). AML is generally recognized as an immunoresponsive malignancy and remains the most common indication for receiving allogeneic hematopoietic stem cell transplantation (11). Previous studies have shown that the suppression of immunity-related antigens in AML cells is correlated with its relapse $(12,13)$. Previous studies showed that immunophenotype is not only useful in the initial diagnosis but also in monitoring and determining prognosis of the disease through minimal residual disease (MRD) testing (14). In a study by Wang et al., the results showed that the abnormal antigen expression of AML is tightly linked with karyotype abnormality. Detection of immunophenotype may help to diagnose and classify AML (15). However, it is still unclear how the expression profile of adaptive immune-related genes affects leukemia development or prognosis.

The current study investigated the expression characteristics and landscape of adaptive immunity-related genes in a previously reported AML population. The subgroups with specific expression patterns were identified and their correlation with survival was assessed. Additionally, we studied the associations of adaptive immunity-related gene expression with clinical features, and evaluated the prognostic roles of individual genes after the detection of differentially expressed genes (DEGs). This work aimed to uncover the novel molecular events in AML and potential therapeutic strategies for AML treatment. We present the following article in accordance with the REMARK reporting checklist (available at http://dx.doi.org/10.21037/atm-21-2720).

\section{Methods}

\section{Patients and RNA-seq}

Normalized RNA sequencing data of 200 patients with AML and their corresponding clinical records were obtained from The Cancer Genome Atlas (TCGA) database (cbioportal.org) (16). Detailed clinical characteristics recorded including patients' age, sex, tumor grade, pathological information, and laboratory test results were collected from the TGCA database. For all 200 patients, AML was diagnosed and validated according to the results of pathological examination. The level of gene expression was expressed as the z-score of messenger RNA (mRNA) and comparisons were conducted between each participant. Due to the lack of related transcriptional information, 27 cases were ruled out of the final analysis, and finally, 173 eligible cases were enrolled into the study. These datasets were publicly available, and ethical approval was granted prior to their use. The study was conducted in accordance with the Declaration of Helsinki (as revised in 2013). 


\section{Bioinformatics}

Similar to previous studies, the present study focused on genes related to adaptive immunity which are statistically annotated in the Kyoto Encyclopedia of Genes and Genomes (KEGG) database (kegg.jp/hsa04514) (17). The profile of 141 genes involved in cell adhesion in AML were assessed, and 8 of them were excluded due to a lack of expression information. To distinguish samples based on their gene expression profile, a cluster analysis of the genetic expression of integral gene sets was performed. Cases with similar gene expression patterns among the entire study population were identified. The transcription levels of related genes were expressed as mRNA z-scores and clustered using a hierarchical clustering algorithm via a Stanford program, as previously described (18). A tumor stage-specific cluster heat map and pattern were generated with Java TreeView (jtreeview.sourceforge.net) (19) and GraphPad Prism (Version 8.0, GraphPad Software, Inc., San Diego, CA, USA).

\section{Prognostic relevance analyses}

The prognostic roles of chromatin remodeling-related genes were investigated through comparison of their survivals between groups with different gene expression levels. The 4 major outcomes included overall survival (OS), progressionfree survival (PFS), disease-free survival (DFS), and diseasespecific survival (DSS). These outcomes were accessed using GraphPad Prism (Version 8.0, GraphPad Software, Inc.). The survival rates of different clusters were compared to reveal the relationship between gene expression levels and prognosis. Additionally, analysis of the difference in OS between cohorts with low or high expression levels of specific genes was conducted in GraphPad Prism (Version 8.0, GraphPad Software, Inc.).

\section{Statistical analysis}

SPSS 24.0 (IBM, Inc., Armonk, NY, USA) was used to carry out statistical analyses. Continuous variables were expressed as mean \pm standard deviation (SD). Categorical variables were expressed as numbers and compared using the $\chi^{2}$ test or Fisher's exact test. Differences in gene expression levels between clusters were detected by analysis of variance (ANOVA). Correlations between variables were determined through regression analyses. Survival curves were plotted and compared between groups using the log-rank test in
GraphPad Prism (Version 8.0, GraphPad Software, Inc.). $\mathrm{P}<0.05$ was considered to indicate statistical significance.

\section{Results}

The expression profile of adaptive immunity-related genes is significantly associated with clinical characteristics of $A M L$

According to hierarchical clustering, patients with AML were grouped into cohorts on the basis of having similar adaptive immunity-related gene expression profile. There were 2 clusters and 3 subclusters identified for all 173 patients, of which cluster 1 and subcluster 1 contained the same patients. The prognosis of each cohort was compared, and no significant difference was apparent in OS or PFS $(\mathrm{P}>0.05)$. Meanwhile, cluster_2 and subcluster_3 showed a slightly worse PFS than the other cohorts, although the difference was not statistically significant ( $\mathrm{P}>0.05)$ (Figure 1A,B, Table 1). Comparison of patients' clinical features revealed that significant differences existed in age, PB blast percentage, and French-AmericanBritish (FAB) classification distribution among both the clusters and subclusters $(\mathrm{P}<0.05)$. Cluster_1/subcluster_1 had a significantly higher average age, a lower PB blast percentage, and more patients with M4 or M5 type (Table 2).

\section{Significant associations exist between adaptive immunity- related gene expression and age and FAB types in $A M L$}

Additionally, we detected the specific differences between each identified cohort. Patients in cluster 1 /subcluster 1 were older than patients in the other subgroups (Figure 2A,B). The dominant FAB types in cluster 1 were M4 (38.64\%) and M5 $(36.36 \%)$, while the dominant FAB types in cluster 2 were M1 (28.68\%) and M2 (26.36\%) (Figure 2C). Compared to subcluster 1, more patients with M4 type and M2 type were included in subcluster 2, and more with M1 type and M2 type were included in subcluster 3 (Figure 2D).

\section{The expression of adaptive immunity-related genes is significantly correlated with age change and $P B$ blast percentage}

We found that cluster_1 had a lower PB blast percentage (26.93 vs. 44.02, $\mathrm{P}=0.002)$ and a higher mean age (60.89 vs. 53.29, $\mathrm{P}=0.006)$ than the cluster_2 (Table 1). To assess the age-associated molecules, the correlation of adaptive 
A

OS of cluster 1 vs. 2

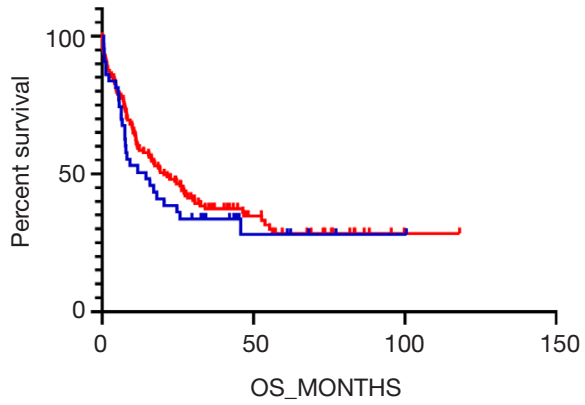

OS of Subcluster 1 vs. 2

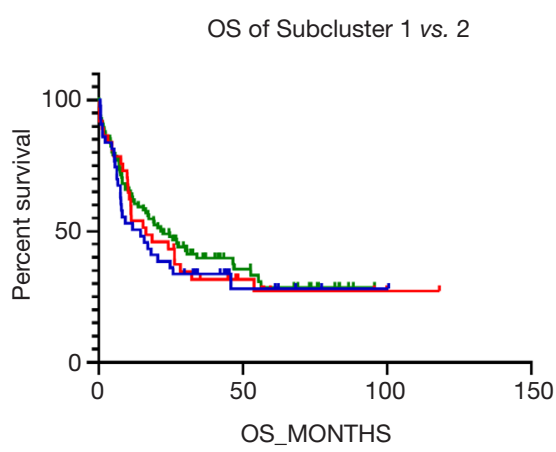

B
PFS of cluster 1 vs. 2

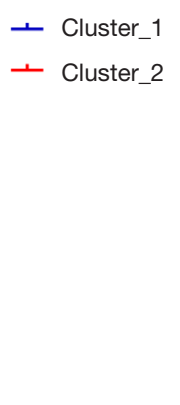

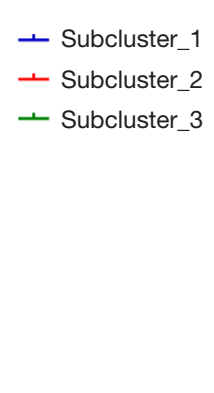

+ Cluster_1

- Cluster_2

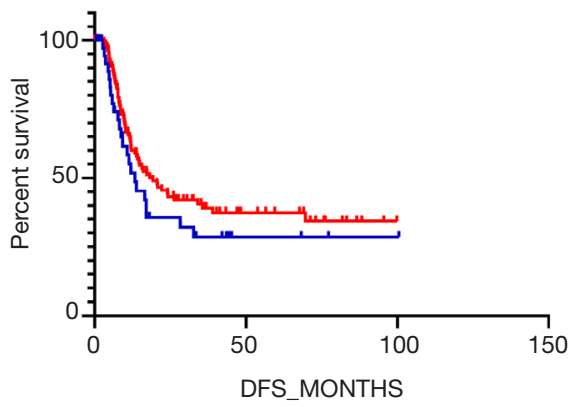

PFS of Subcluster 1 vs. 2

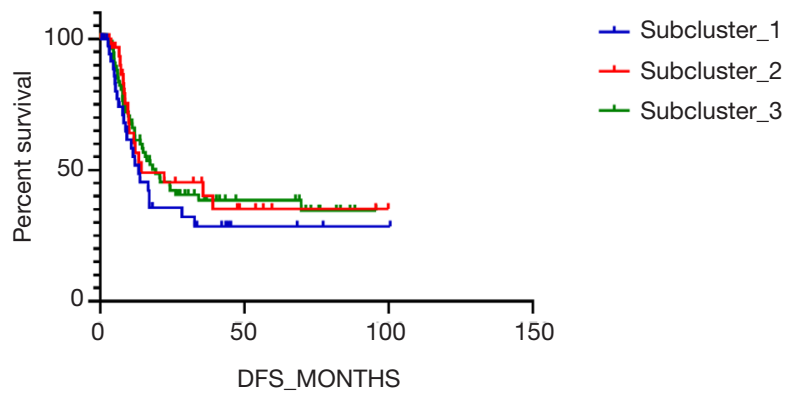

Figure 1 The expression profile of adaptive immunity-related genes is significantly associated with AML clinical characteristics. An insignificant difference in overall survival (OS) and progression-free survival (PFS) between the clusters (A) and subclusters $(\mathrm{B})(\mathrm{P}>0.05)$.

Table 1 Survival differences in the cohorts

\begin{tabular}{lccccc}
\hline Identified cohort & OS & Hazard ratio (95\% Cl of ratio) & P value & PFS & Hazard ratio (95\% Cl of ratio) \\
\hline Cluster_1 & 14.5 & $1.176(0.757-1.827)$ & 0.449 & 13.4 & $1.355(0.806-2.277)$ \\
Cluster_2 & 20.5 & & & 19.3 & 0.214 \\
Subcluster_1 & 14.5 & & 0.687 & 13.4 & 0.461 \\
Subcluster_2 & 16.4 & & & 14.2 & 19.3 \\
Subcluster_3 & 22.3 & & & & \\
\hline
\end{tabular}

OS, overall survival; PFS, progression-free survival.

immunity-related gene expression with age was evaluated. There were 81 genes markedly correlated with age, including 61 positively correlated genes and 20 negatively correlated genes (Figure 3A). Among these age-associated molecules, F-box protein 32 (FBXO32), cytotoxic T-lymphocyte-associated protein 4 (CTLA4), and killer cell lectin-like receptor F1 (KLRF1) had the strongest positive correlations, and ORAI calcium release-activated calcium modulator 2 (ORAI2), cell division cycle 23 (CDC23), and cluster of differentiation 96 (CD96) had the strongest negative correlations. Assessment of the relationship of adaptive immunity-related genes with PB blast percentage showed that there were 101 genes notably correlated with PB blast percentage, including 6 positively correlated genes and 95 negatively correlated genes (Figure 3B). Among these $\mathrm{PB}$ blast percentage-associated molecules, zinc and ring finger 1 (ZNRF1), ubiquitin-conjugating enzyme E2 Z (UBE2Z), and cluster of differentiation 99 (CD99) had the strongest positive correlations, and tubulin alpha $4 \mathrm{~A}$ (TUBA4A), cathepsin H (CTSH), and protein tyrosine phosphatase receptor type $\mathrm{J}$ (PTPRJ) had the strongest negative correlations. 
Table 2 Comparison of clinical features between each cluster or subcluster

\begin{tabular}{|c|c|c|c|c|c|c|c|}
\hline Clinical features & $\begin{array}{l}\text { Cluster_1 } \\
(n=44)\end{array}$ & $\begin{array}{c}\text { Cluster_2 } \\
(n=129)\end{array}$ & $P$ value & $\begin{array}{l}\text { Subcluster_1 } \\
\qquad(n=44)\end{array}$ & $\begin{array}{l}\text { Subcluster_2 } \\
\qquad(n=37)\end{array}$ & $\begin{array}{l}\text { Subcluster_3 } \\
\qquad(\mathrm{n}=92)\end{array}$ & $P$ value \\
\hline Age & $60.89 \pm 13.41$ & $53.29 \pm 16.59$ & 0.007 & $60.89 \pm 13.41$ & $55.05 \pm 13.92$ & $52.59 \pm 17.57$ & 0.019 \\
\hline \multicolumn{8}{|l|}{ Sex } \\
\hline Female & 24 & 57 & 0.294 & 24 & 13 & 44 & 0.210 \\
\hline WBC & $45.18 \pm 38.08$ & $33.72 \pm 48.30$ & 0.155 & $45.18 \pm 38.08$ & $35.12 \pm 44.66$ & $33.15 \pm 49.92$ & 0.356 \\
\hline PB blast percentage & 26.93 & 44.02 & 0.002 & 26.93 & 35.67 & 47.36 & 0.002 \\
\hline \multicolumn{8}{|l|}{ FAB type } \\
\hline M3 & 0 & 16 & & 0 & 1 & 15 & \\
\hline M4 & 17 & 17 & & 17 & 12 & 5 & \\
\hline M5 & 16 & 2 & & 16 & 1 & 1 & \\
\hline M6 & 0 & 2 & & 0 & 1 & 1 & \\
\hline M7 & 0 & 3 & & 0 & 1 & 2 & \\
\hline NC & 0 & 2 & & 0 & 1 & 1 & \\
\hline
\end{tabular}

BM, bone marrow; WBC, white blood cell; PB, peripheral blood; FAB, French-American-British.

\section{The expression of adaptive immunity-related genes is correlated with AML FAB types}

Since the distributions of FAB subtypes differed significantly for each gene expression pattern, we next studied the correlation of adaptive immunity-related gene expression with FAB types. We compared OS and PFS between patients with each FAB type and uncovered those patients with M3 type showed the most favorable outcome (Figure 4A). Assessment of DEGs between M3 type and the other types showed that 306 genes were significantly varied $(\mathrm{P}<0.05)$ and functionally related (Figure $4 B)$. KEGG functional enrichment analysis of these genes revealed that phagosome function, antigen processing and presentation, and ubiquitin-mediated proteolysis were the 3 most significant functional sets among DEGs in patients with M3 type (Figure 4C). Among the DEGs, C-type lectin domain family 2 member B (CLEC2B) showed a significantly low expression level in $\mathrm{M} 3$ type and a high expression in the other types (Figure 4D). Moreover, high expression of CLEC2B indicated an unfavorable OS of AML (12.40 vs.
46.50 months, $\mathrm{P}=0.003$ ) (Figure 4E).

\section{Discussion}

Variation in adaptive immunity-related gene expression is significantly associated with clinical characteristics and relapse in patients with AML. Therefore, illuminating the landscape of adaptive immunity-related gene expression can greatly facilitate the understanding of AML. For example, some studies showed that CD25 is an independent prognostic factor in AML patients and alternative therapies for CD25-positive AML patients are needed (20). In the present work, we assessed an expression profile containing 732 genes and grouped 173 patients into different cohorts according to the expression pattern. Comparison of outcomes among the patients showed that the identified cluster_1 (or subcluster_1) had a shorter OS (14.5 vs. 20.5 months) and PFS (13.4 vs. 19.3 months). Nevertheless, no statistically significant difference was detected $(\mathrm{P}>0.05)$. This negative result might be attributable to the limited 
A

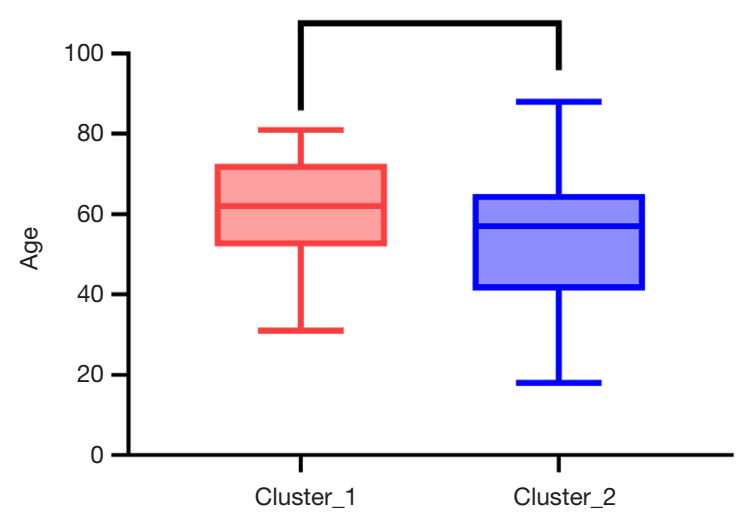

B

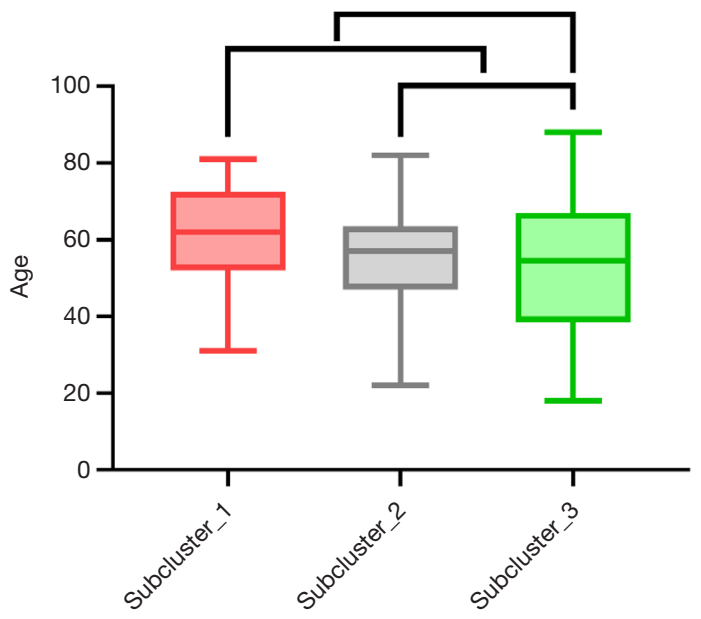

C

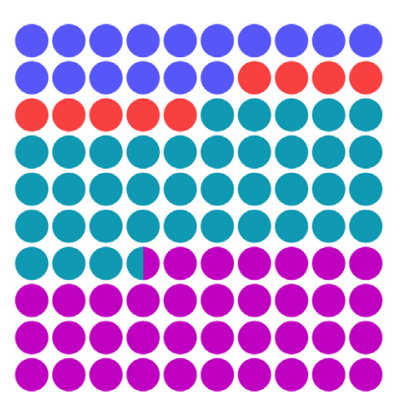

Total $=44$



Total $=129$

D
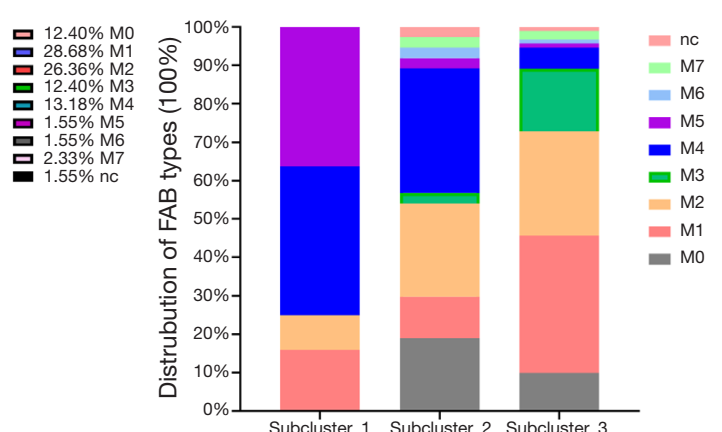

Figure 2 The subgroups according to adaptive immunity-related gene expression are significantly associated with age and FAB type in AML. (A,B) Patients in cluster 1 and subcluster 1 were older than patients in other subgroups; (C) the dominant FAB types in cluster 1 were M4 (38.64\%) and M5 (36.36\%), while in cluster 2, the dominant FAB types were M1 (28.68\%) and M2 (26.36\%); (D) compared to subcluster 1, more patients with M4 type and M2 type were included in subcluster 2, and more patients with M1 type and M2 type were included in subcluster 3. *, $\mathrm{P}<0.05$; ${ }^{* *}, \mathrm{P}<0.01$. FAB, French-American-British; AML, acute myeloid leukemia.

sample size. Regarding clinical characteristics, patients in cluster_1 were older on average (60.89 vs. 53.29 years), which supported cluster_1 having a worse prognosis. PB blast percentage and blood marrow blast percentage are considered to be important prognostic factors for AML, along with the relative ratio $(21,22)$. According to our data, no difference was detected in the BM blast percentage between each cluster or subcluster. However, cluster_1 was associated with a remarkably lower PB blast percentage than the other clusters.

To analyze the correlations of adaptive immunityrelated gene expression with age and $\mathrm{PB}$ blast percentage, we performed correlation analyses. There were 81 adaptive immunity-related genes that were obviously correlated with age including 61 positively correlated genes and 20 negatively correlated genes. A relationship between differences in adaptive immunity-related genes and older age was revealed. There were 101 genes that were correlated with PB blast percentage. Interestingly, among these PB blast percentage-associated genes, only 6 genes were positively correlated with increased PB blast percentage. The expression of most PB blast percentageassociated genes was negatively correlated with increased PB blast percentage. These results suggest that expression differences in adaptive immunity-related genes are closely associated with age and PB blast percentage in AML.

We uncovered that the distribution of FAB subtypes differed significantly between the cohorts identified based on adaptive immunity-related gene expression. In cluster 1, 
A

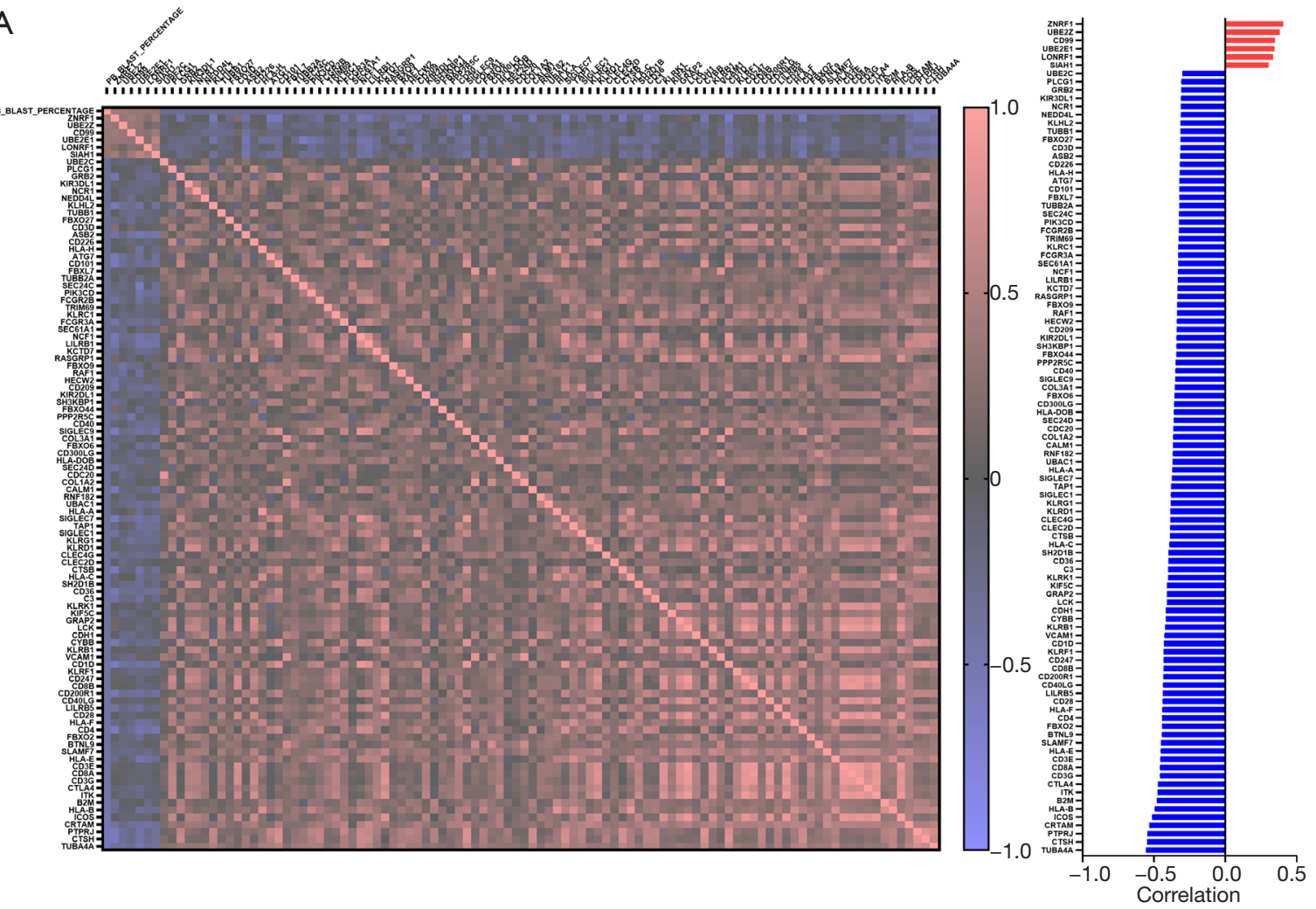

B

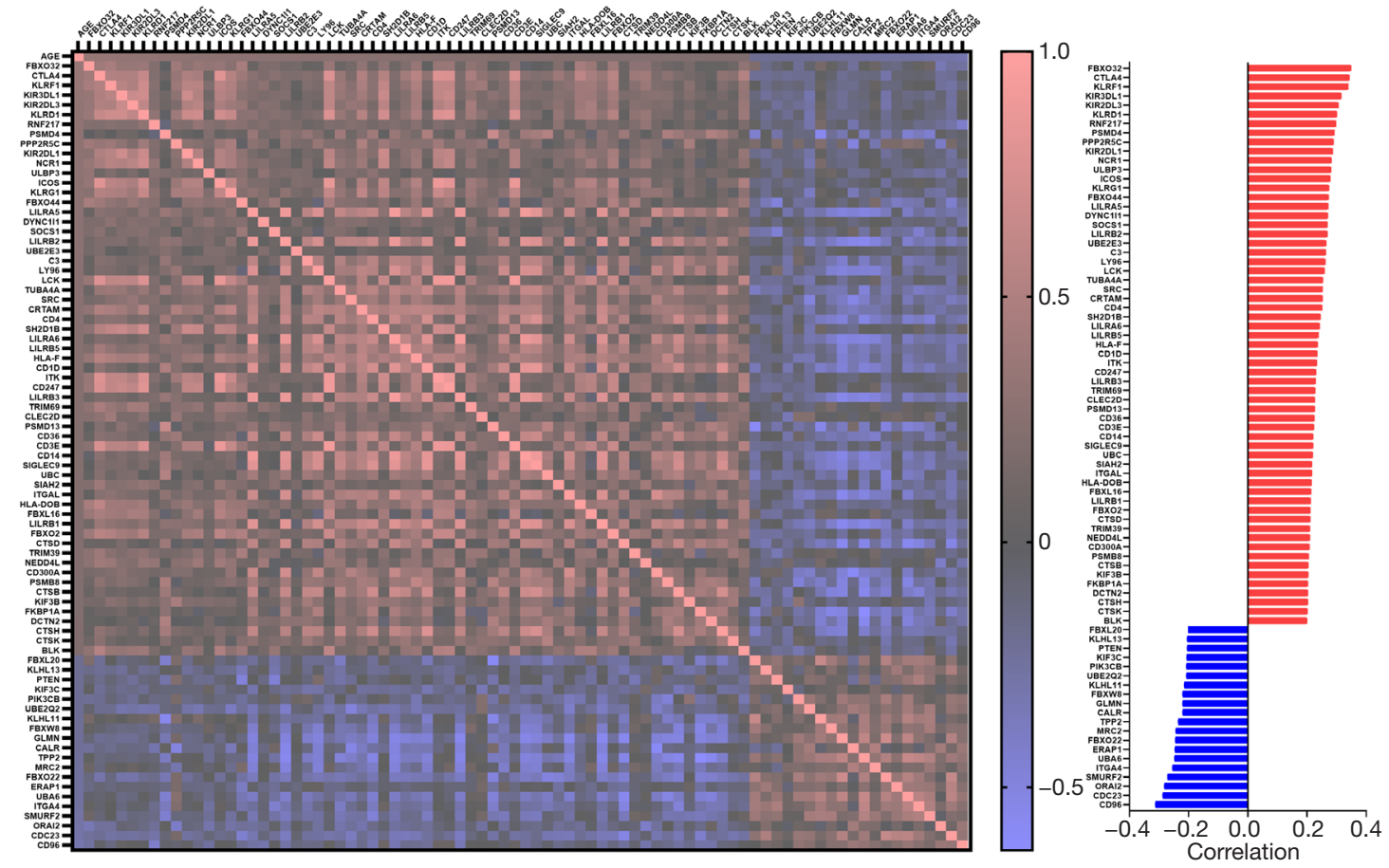

Figure 3 Adaptive immunity-related gene expression was significantly correlated with age and peripheral blood blast percentage. (A) There were 81 genes markedly correlated with age, including 61 positively correlated genes and 20 negatively correlated genes; (B) there were 101 genes markedly correlated with PB blast percentage, including 6 positively correlated genes and 95 negatively correlated genes. PB, peripheral blood. 
A

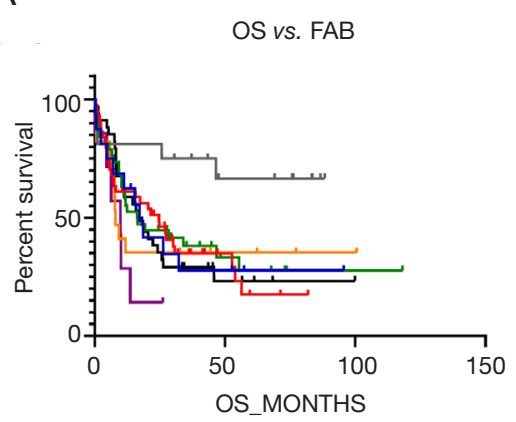

PFS vs. FAB



B
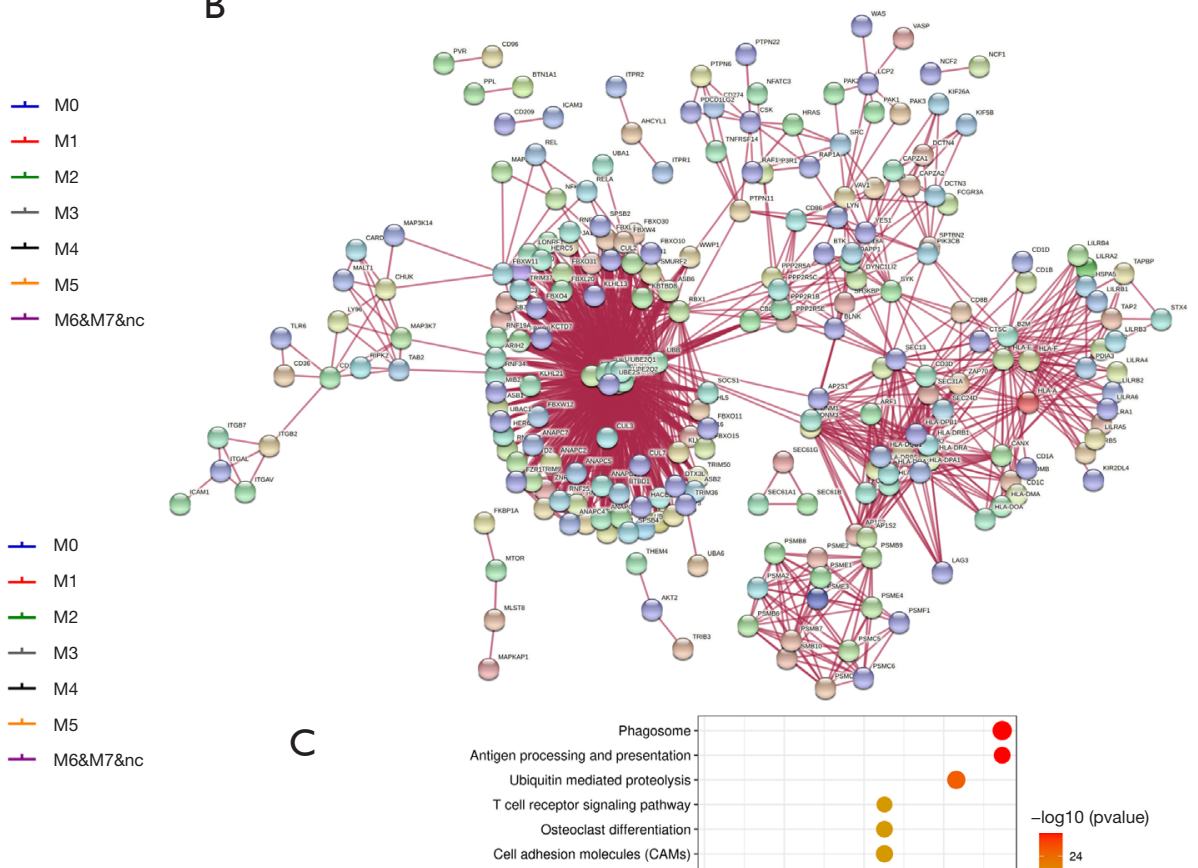

C

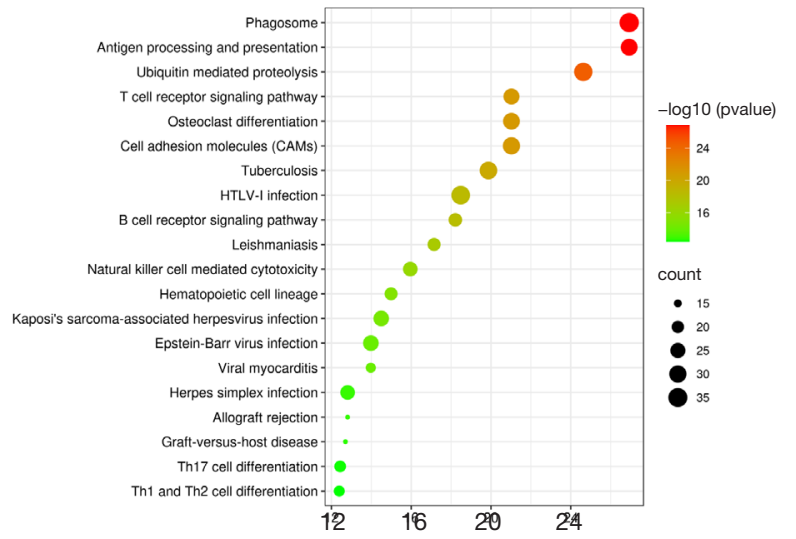

D

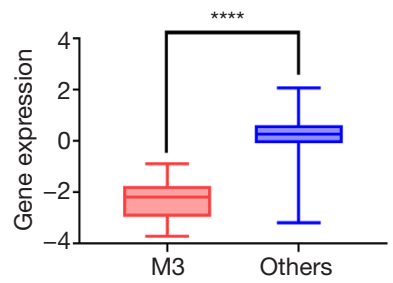

$\mathrm{E}$

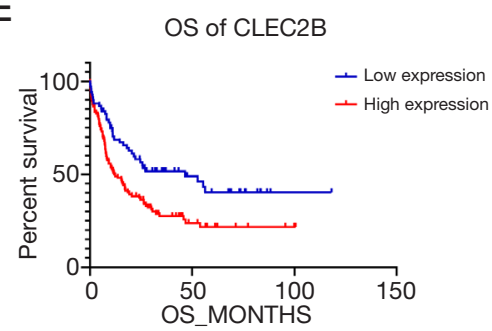
Figure 4 Expression of adaptive immunity-related genes was correlated with AML FAB types. (A) Patients with M3 type showed the
most favorable outcome; (B) 306 genes were significantly varied and functionally related; (C) phagosome function, antigen processing and presentation, and ubiquitin-mediated proteolysis were the 3 most significant functional sets among DEGs in the M3 type; (D) CLEC2B showed a significantly low expression level in M3 type but was highly expressed in the other types. (E) high expression of CLEC2B was significantly correlated to an unfavorable overall survival of AML. ${ }^{* * *}, \mathrm{P}<0.0001$. FAB, French-American-British; AML, Acute myeloid leukemia; DEGs, differentially expressed genes.

the most widely distributed FAB types were M4 and M5, while in cluster 2, M1 and M2 were dominantly distributed. These data indicated a significant correlation between adaptive immunity and FAB subtypes. Previous studies have shown that gene aberrations were closely related to the distribution of FAB types and associated with AML prognosis (23). In the current work, we found that patients with M3 type exhibited a prolonged OS and PFS compared to patients with non-M3 types, which is consistent with the results of a previous report (24). Additional assessment of the difference in adaptive immunity-related gene expression between $\mathrm{M} 3$ type and non-M3 types indicated that CLEC2B expression differed significantly. In patients with the M3 type, CLEC2B expression was low, while in patients with non-M3 types, it was significantly elevated. Moreover, the high expression of CLEC2B was associated with a worse OS in patients with AML. CLEC-2 is activated by the transmembrane protein podoplanin, which is found outside of the vasculature and is upregulated in development, inflammation, and cancer, and there is also evidence for additional ligands (25). In melanoma, CLEC2B was reported to be highly expressed in melanoma tissues 
and associated with a poor prognosis (26). Together, these results suggest that CLEC2B has the potential to serve as a prognostic marker for predicting the survival of AML.

In conclusion, our study has demonstrated that adaptive immunity-related genes showed significant expression differences in AML, and the expression patterns were closely associated with age, FAB type, and PB blast percentage in patients with AML. CLEC2B was specifically lowly expressed in patients with M3 type, and its high expression predicted a poor outcome; therefore, it might be pursued as a prognostic factor and treatment target. As for limitation, the results of our present study need to be further investigated in clinical trials in the future.

\section{Acknowledgments}

Funding: None.

\section{Footnote}

Reporting Checklist: The authors have completed the REMARK reporting checklist. Available at http://dx.doi. org/10.21037/atm-21-2720

Conflicts of Interest: All authors have completed the ICMJE uniform disclosure form (available at http://dx.doi. org/10.21037/atm-21-2720). The authors have no conflicts of interest to declare.

Ethical Statement: The authors are accountable for all aspects of the work in ensuring that questions related to the accuracy or integrity of any part of the work are appropriately investigated and resolved. The study was conducted in accordance with the Declaration of Helsinki (as revised in 2013).

Open Access Statement: This is an Open Access article distributed in accordance with the Creative Commons Attribution-NonCommercial-NoDerivs 4.0 International License (CC BY-NC-ND 4.0), which permits the noncommercial replication and distribution of the article with the strict proviso that no changes or edits are made and the original work is properly cited (including links to both the formal publication through the relevant DOI and the license). See: https://creativecommons.org/licenses/by-nc-nd/4.0/.

\section{References}

1. Ehninger A, Kramer M, Röllig C, et al. Distribution and levels of cell surface expression of CD33 and CD123 in acute myeloid leukemia. Blood Cancer J 2014;4:e218.

2. Christopher MJ, Petti AA, Rettig MP, et al. Immune Escape of Relapsed AML Cells after Allogeneic Transplantation. N Engl J Med 2018;379:2330-41.

3. Mielcarek M, Storer BE, Flowers ME, et al. Outcomes among patients with recurrent high-risk hematologic malignancies after allogeneic hematopoietic cell transplantation. Biol Blood Marrow Transplant 2007;13:1160-8.

4. Papaemmanuil E, Gerstung M, Bullinger L, et al. Genomic Classification and Prognosis in Acute Myeloid Leukemia. N Engl J Med 2016;374:2209-21.

5. Arndt C, von Bonin M, Cartellieri M, et al. Redirection of T cells with a first fully humanized bispecific CD33-CD3 antibody efficiently eliminates AML blasts without harming hematopoietic stem cells. Leukemia 2013;27:964-7.

6. Angelini DF, Ottone T, Guerrera G, et al. A LeukemiaAssociated CD34/CD123/CD25/CD99+ Immunophenotype Identifies FLT3-Mutated Clones in Acute Myeloid Leukemia. Clin Cancer Res 2015;21:3977-85.

7. Cerny J, Yu H, Ramanathan M, et al. Expression of CD25 independently predicts early treatment failure of acute myeloid leukaemia (AML). Br J Haematol 2013;160:262-6.

8. Chang H, Yeung J, Brandwein J, et al. CD7 expression predicts poor disease free survival and post-remission survival in patients with acute myeloid leukemia and normal karyotype. Leuk Res 2007;31:157-62.

9. Vesely MD, Kershaw MH, Schreiber RD, et al. Natural innate and adaptive immunity to cancer. Annu Rev Immunol 2011;29:235-71.

10. Haseeb M, Anwar MA, Choi S. Molecular Interactions Between Innate and Adaptive Immune Cells in Chronic Lymphocytic Leukemia and Their Therapeutic Implications. Front Immunol 2018;9:2720.

11. Vago L, Gojo I. Immune escape and immunotherapy of acute myeloid leukemia. J Clin Invest 2020;130:1552-64.

12. Vago L, Perna SK, Zanussi M, et al. Loss of mismatched HLA in leukemia after stem-cell transplantation. $\mathrm{N}$ Engl J Med 2009;361:478-88.

13. Vadakekolathu J, Minden MD, Hood T, et al. Immune landscapes predict chemotherapy resistance and immunotherapy response in acute myeloid leukemia. Sci Transl Med 2020;12:eaaz0463.

14. Galera PK, Jiang C, Braylan R. Immunophenotyping of Acute Myeloid Leukemia. Methods Mol Biol 2019;2032:281-96.

15. Wang XB, Zheng JE, Gu JX, et al. Correlation of 
immunophenotype to cytogenetics and clinical features of adult acute myeloid leukemia. Ai Zheng 2005;24:667-71.

16. Gao J, Aksoy BA, Dogrusoz U, et al. Integrative analysis of complex cancer genomics and clinical profiles using the cBioPortal. Sci Signal 2013;6:pl1.

17. Zhang Z, Yu H, Jiang S, et al. Evidence for Association of Cell Adhesion Molecules Pathway and NLGN1 Polymorphisms with Schizophrenia in Chinese Han Population. PLoS One 2015;10:e0144719.

18. de Hoon MJ, Imoto S, Nolan J, et al. Open source clustering software. Bioinformatics 2004;20:1453-4.

19. Saldanha AJ. Java Treeview--extensible visualization of microarray data. Bioinformatics 2004;20:3246-8.

20. Fujiwara SI, Muroi K, Yamamoto C, et al. CD25 as an adverse prognostic factor in elderly patients with acute myeloid leukemia. Hematology 2017;22:347-53.

21. Amin HM, Yang Y, Shen Y, et al. Having a higher blast percentage in circulation than bone marrow: clinical implications in myelodysplastic syndrome and acute lymphoid and myeloid leukemias. Leukemia 2005;19:1567-72.

Cite this article as: Chen P, Liu Y, Zhang R, Wang H, Zhang J, Guo M, Du Z. Adaptive immunity-related gene expression profile is correlated with clinical phenotype in patients with acute myeloid leukemia. Ann Transl Med 2021;9(11):939. doi: 10.21037/atm-21-2720
22. Zeijlemaker W, Kelder A, Oussoren-Brockhoff YJ, et al. Peripheral blood minimal residual disease may replace bone marrow minimal residual disease as an immunophenotypic biomarker for impending relapse in acute myeloid leukemia. Leukemia 2016;30:708-15.

23. Noort S, Zimmermann M, Reinhardt D, et al. Prognostic impact of $\mathrm{t}(16 ; 21)(\mathrm{p} 11 ; \mathrm{q} 22)$ and $\mathrm{t}(16 ; 21)(\mathrm{q} 24 ; \mathrm{q} 22)$ in pediatric AML: a retrospective study by the I-BFM Study Group. Blood 2018;132:1584-92.

24. de Lima MC, da Silva DB, Freund AP, et al. Acute Myeloid Leukemia: analysis of epidemiological profile and survival rate. J Pediatr (Rio J) 2016;92:283-9.

25. Rayes J, Watson SP, Nieswandt B. Functional significance of the platelet immune receptors GPVI and CLEC-2.J Clin Invest 2019;129:12-23.

26. Gao Y, Li Y, Niu X, et al. Identification and Validation of Prognostically Relevant Gene Signature in Melanoma. Biomed Res Int 2020;2020:5323614.

(English Language Editor: J. Reynolds) 